\title{
Quality of life in inflammatory bowel diseases: it is not all about the bowel
}

\author{
Ronald Keller ${ }^{1}$, Nazar Mazurak ${ }^{2}$, Laura Fantasia ${ }^{1}$, Stefano Fusco ${ }^{1}$, Nisar P Malek ${ }^{1}$, Jan Wehkamp ${ }^{1}$, Paul Enck ${ }^{2}$, \\ Thomas Klag ${ }^{1}$ \\ ${ }^{I}$ Division of Gastroenterology, Hepatology, Infectious Diseases, Department of Internal Medicine I and ${ }^{2}$ Division of Psychosomatic Medicine and \\ Psychotherapy, Department of Internal Medicine VI, University Hospital Tübingen, Tübingen, Germany
}

Background/Aims: The inflammatory bowel diseases (IBD), ulcerative colitis (UC), and Crohn's disease (CD) are chronic diseases mostly affecting young patients. As they are diseases accompanying patients for their entire life, and the quality of life (QUOL) interacts with disease activity, improving QUOL should be one of the main goals of therapy. This study aims to identify factors contributing to good or impaired QUOL. Methods: Questionnaires addressing health-related QUOL and other psychological and social features were positioned on our institutions' webpage and on the webpage of the largest self-help group for IBD in Germany. Patients were subdivided according to their QUOL score with a cutoff of $<60$. We used the Short Inflammatory Bowel Disease Questionnaire, the Assessment of the Demand for Additional Psychological Treatment, and the Fear of Progression Questionnaire Short Form. Results: High numbers of patients in both subgroups showed an impaired QUOL (87.34\% in UC, 91.08\% in CD). Active extraintestinal manifestations, smoking, high fear of progression and high demand for psychotherapy were associated with reduced QUOL. In addition, polypharmacological interventions did not result in a good QUOL, but ostomies are linked to improved QUOL especially in CD patients. Conclusions: Scores used in clinical day-to-daypractice mainly focusing on somatic factors do not sufficiently address important aspects concerning QUOL. Most importantly, extraintestinal manifestations show a hitherto underestimated impact on QUOL. (Intest Res 2021;19:45-52)

Key Words: Quality of life; Crohn disease; Colitis, ulcerative; Extraintestinal manifestations

\section{INTRODUCTION}

The inflammatory bowel disease (IBD), Crohn's disease (CD), and ulcerative colitis (UC) affect ca. 300-400 thousand patients in Germany, showing a prevalence of about $1 \%$ in industrialized countries. ${ }^{1,2}$ As these are chronic diseases that are often diagnosed at a relatively young age, thus accompanying patients for a long time, and have great impact on their course of life, a good quality of life (QUOL) should be among the main therapeutic goals.

Received October 21, 2019. Revised November 27, 2019.

Accepted December 23, 2019.

Correspondence to Thomas Klag, Department of Internal Medicine I,

University Hospital Tübingen, Otfried-Müller-Str. 10, 72076 Tübingen,

Germany. Tel: +49-7071-29-0, Fax: +49-7071-29-2095, E-mail: thomas.

klag@med.uni-tuebingen.de
Although disease activity scores exist that include the QUOL, they are often not validated and are laborious to use, pushing clinicians to relying on endoscopic or simpler scores with a lower emphasis on QUOL. ${ }^{3}$ In addition, most scores (that are also utilized for the assessment of therapeutic response as primary end points in pivotal studies for IBD-medications ${ }^{4}$ ) mainly focus on intestinal symptoms such as e.g. hematochezia, stool frequency and abdominal pain, although IBD often concur with extraintestinal manifestations (EIM), such as painful arthritis and a variety of other manifestations, e.g. skin diseases and eye affections, ${ }^{5}$ resulting in increased morbidity and likely to a reduced QUOL.

Therefore, investigating the influence of these non-bowel related symptoms on QUOL could be helpful in managing IBD in daily care. In these lines, in a patient perspective, one fur- 
ther milestone of IBD may be the creation of a stoma, ${ }^{6}$ which has a great impact on lifestyle and may thereby reduce QUOL, while on the other hand may bring great relief to patients by allowing some more control of bowel movements.

Another environmental influence on IBD is smoking, which has already been shown to impact disease onset and progression: CD patients who smoke have an increased risk of recurrence after surgery and poor response to medical therapy, while individuals with UC appear to profit from a protective effect of smoking on their course of disease. ${ }^{7}$ However, these data are also based on disease activity scores rather than QUOL scores.

The primary goal of this study is the evaluation of a set of non-intestinal social, clinical and psychometric test variables associated with QUOL to develop an improved test battery to monitor patients QUOL during the course of disease, as well as to identify contributors to superior QUOL that to date may not be addressed sufficiently in daily care. For the generation of a sufficiently big patient cohort without physician-driven selection that may distort our results we set out to explore the QUOL of unselected patients with either UC or CD, independent of their current disease management (primary, secondary, and tertiary centers), using a web-based questionnaire.

\section{METHODS}

For 14 months, a questionnaire with general questions and psychological scores was placed on the webpage of our institution that was freely accessible to all interested patients. Furthermore, the questionnaire was announced on the website of the largest German self-help group, the DCCV, “Deutsche Morbus Crohn/Colitis ulcerosa Vereinigung e.V." (DCCV e.V.; www. dccv.de), and mentioned in newsletters sent to all DCCV members $(n=20,667)$. Patients with IBD seen at our hospital were also asked to complete the online survey. The study was approved by the Ethics Board of the University Medical School of Tübingen (approval No. 12112014). Written informed consents were obtained from all patients included.

\section{Questionnaire-Bio-Psychosocial Factors}

The questionnaire included questions assessing disease history (diagnosis, disease duration, intestinal and EIM, treatments including surgery/medication), social data (age and sex), and health behaviors (smoking and DCCV membership).

\section{Short Inflammatory Bowel Disease Questionnaire} The Short Inflammatory Bowel Disease Questionnaire (SIBDQ) is a 10 -item questionnaire (issues rated between 0 and 7$)^{8-10}$ that has been validated against other QUOL measures and against its original version, the Inflammatory Bowel Disease Questionnaire (IBDQ). ${ }^{11}$ The validated German version is available since $2000 .{ }^{12}$ The score ranges between 10 and 70 points. QUOL is considered to be slightly (60-70 points), moderately (45-60 points) or severely impaired (10-45 points). ${ }^{13}$ Therefore, in our cohort, we defined the cut off for relevant impairment of QUOL $<60$ points.

\section{Assessment of the Demand for Additional Psychological Treatment}

The Assessment of the Demand for Additional Psychological Treatment (ADAPT) is a 12-item German-language questionnaire, with every question answered on visual analog scale between 0 ("no, not at all") to 100 ("yes, completely right"). ${ }^{14} \mathrm{~A}$ summarized total score of $>60$ indicates increased demand for psychological support. The score has been investigated in patients with $\mathrm{IBD}^{14-16}$ but also to patients with other chronic conditions. ${ }^{17,18}$ We have used it to explore the demand of psychotherapy in this cohort.

\section{Fear of Progression Questionnaire Short Form}

The Fear of Progression Questionnaire Short Form (FoP-Q$\mathrm{SF}$ ) rates 12 items between 0 (never) and 4 (very often) on a 5-point Likert-scale that are added for a total score. ${ }^{19}$ The score includes different aspects of anxiety in daily life affected by chronic diseases. Fear of progression (FoP) has been studied in cancer and other chronically ill patients, e.g., patients with rheumatoid arthritis, where high values were found. ${ }^{20}$

\section{Statistics}

Only complete data sets were analyzed. Patients were subdivided according to their SIBDQ score with a cutoff of $\geq 60$ and $<60$ (yes, no). Subsequently, qualitative data were tested between groups by chi-square tests, and numeric data and psychometric test scores were entered into a two-way analysis of variance. Relevant data (that were identified in the previous analyses) were then entered into a multiple regression analysis with ordinal and nominal variables (age, sex, smoking, diagnosis [CD, UC], duration of disease, DCCV membership, any previous surgery and EIMs) as variables to be used in a stepwise forward manner. Regression was analyzed for QUOL (SIBDQ) using the FoP-Q-SF score and the ADAPT score as additional potential predictors. A stepwise forward inclusion was used to find the model with maximum R2 that has the 
highest explanation potential for all IBD patients together. The analysis was repeated for patients with UC and CD separately. All statistics were performed using SPSS version 19 (IBM Corp., Armonk, NY, USA). All data are reported as mean \pm standard deviation, and the alpha level indicating statistical significance was set at $<0.05$.

\section{RESULTS}

\section{Study Population}

A total of 631 individuals responded to the questionnaire, resulting in 578 complete data sets that were used for the further analysis. Detailed patient characteristics are summarized in Supplementary Table 1. In summary, 36.7 years was the mean

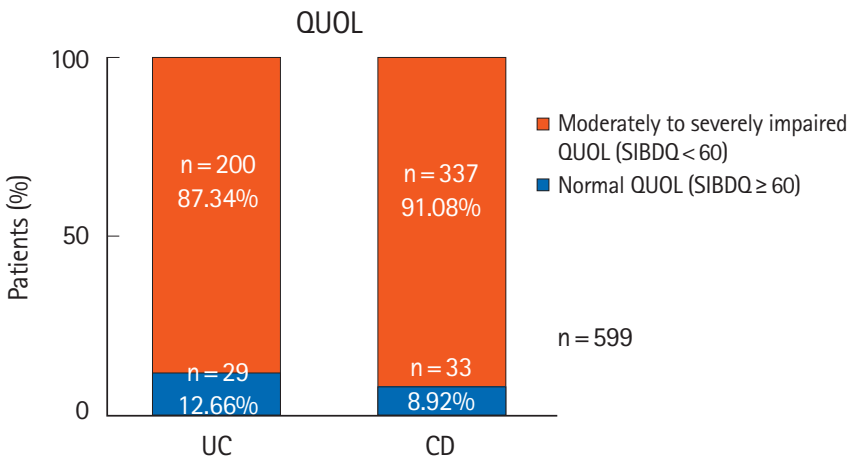

Fig. 1. Quality of life (QUOL) in patients with CD and UC (whole cohort). QUOL is reduced in the majority of patients, but do not differ significantly between IBD-entities. A Short Inflammatory Bowel Disease Questionnaire (SIBDO) score $<60$ is considered as moderate to severe impairment of QUOL. CD, Crohn's disease; UC, ulcerative colitis; IBD, inflammatory bowel disease.

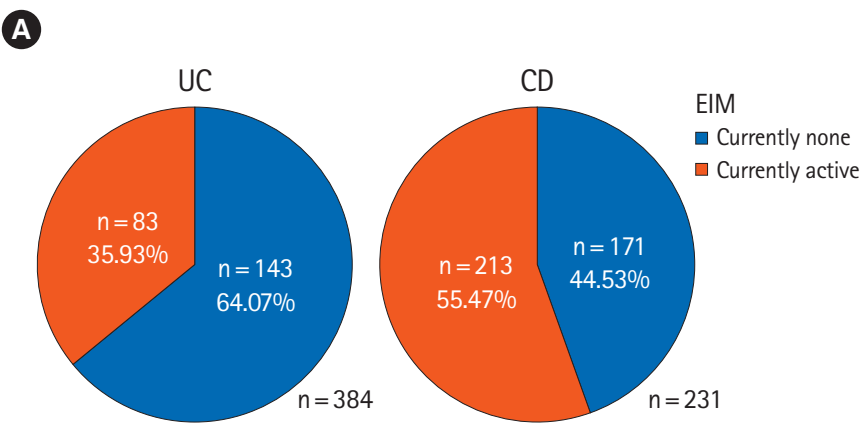

age, and more women responded to the survey ( $75 \%$ female vs. $25 \%$ male). Over $50 \%$ of the respondents suffered from IBD for more than 10 years.

\section{QUOL in Patients with IBD}

Initially, we analyzed the data regarding patients' QUOL, with a SIBDQ score $<60$ defined as moderately to severe impaired QUOL and $\geq 60$ as normal QUOL. A total of 200 patients (87.34\%) suffering from UC and 337 patients (91.08\%) suffering from CD showed an impaired QUOL, yielding no significant difference between both entities (Fig. 1).

Analyzing the burden of EIM in our cohort we found that 83 (35.93\%) of UC and 213 (55.47\%) of CD patients suffer from active EIMs. Thus, we analyzed the impact of EIM on patients QUOL. Our data yielded a significant negative impact of EIM on QUOL, with a median SIBDQ score of 47.1 without and 38.9 with active EIM, showing no significant difference between both entities (Fig. 2).

In total, $9.17 \%$ of our UC patients and $16.45 \%$ of CD patients have or had a stoma at the time of our investigation. As the creation of a stoma has a deep impact on lifestyle during the course of IBD, we analyzed its impact on QUOL. Interestingly, the QUOL of CD patients who currently live with a stoma is significantly improved in comparison with patients without stoma and who used to have a stoma, but had it reversed (Fig. $3)$. However, IBD surgery in general during the course of disease did not show a significant effect on QUOL (Table 1). Furthermore, in UC a stoma had no impact on QUOL in our cohort (Fig. 3).

Analyzing some health behavior-related issues regarding

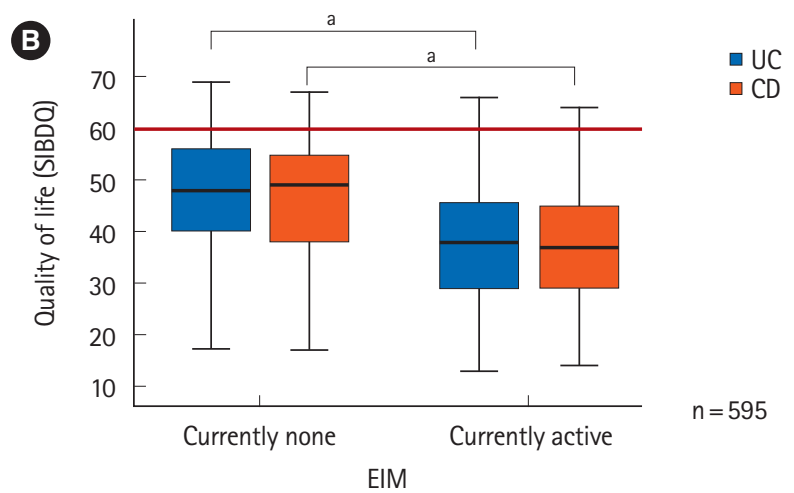

Fig. 2. Extraintestinal manifestations (EIM) are significantly associated with a reduced quality of life (QUOL). (A) $35.93 \%$ (UC) to $55.47 \%$ (CD) of IBD patients suffer from active EIM. (B) QUOL is significantly reduced in patients with active EIM in both entities. The boxplot's median is depicted using a horizontal black line. Cutoff of the score for the QUOL was 60 points in the Short Inflammatory Bowel Disease Questionnaire (SIBDO) and is depicted using a red line ( $\geq 60$ normal QUOL, $<60$ moderately to severely reduced QUOL). A $t$-test was used for statistical analysis. ${ }^{a} P<0.001$. UC, ulcerative colitis; $C D$, Crohn's disease; IBD, inflammatory bowel disease. 

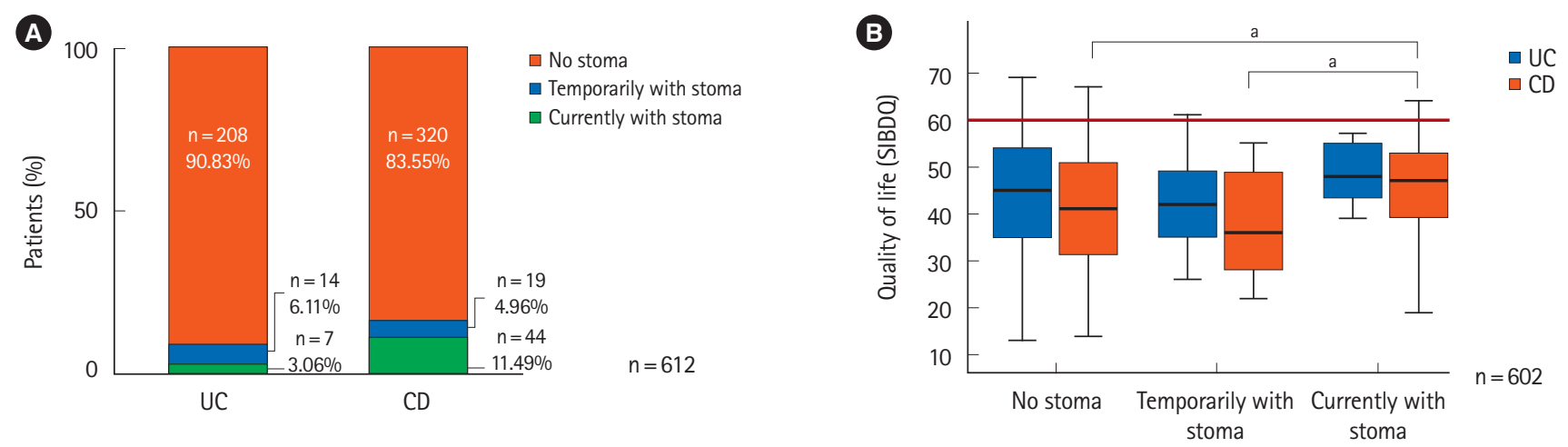

Fig. 3. Influence of abdominal stoma on quality of life (QUOL). (A) 9.17\% (UC) to $16.45 \%$ (CD) of the IBD patients in our cohort have or had a stoma application during their course of disease. (B) QUOL of CD patients can be improved by stoma application, while this procedure does not show an effect on QUOL in UC patients in our cohort. The boxplot's median is depicted using a horizontal black line. Cutoff of the score for the QUOL was 60 points in the Short Inflammatory Bowel Disease Questionnaire (SIBDO) and is depicted using a red line ( $\geq 60$ normal QUOL, $<60$ moderately to severely reduced QUOL). A $t$-test was used for statistical analysis. ${ }^{a} P<0.05$. UC, ulcerative colitis; $C D$, Crohn's disease; IBD, inflammatory bowel disease.
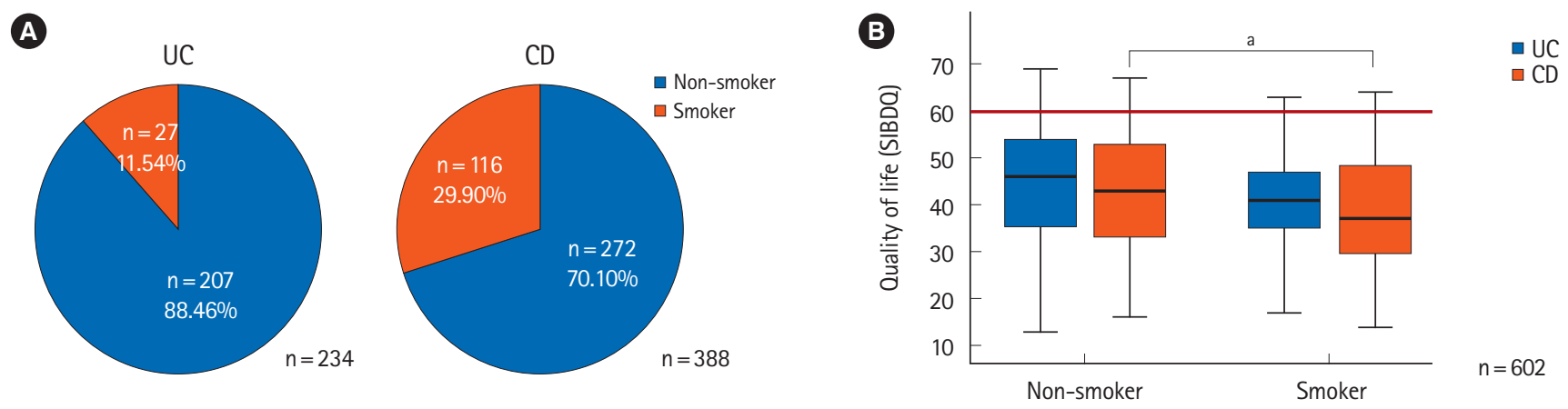

Fig. 4. Smoking behavior and its influence on quality of life (QUOL) in IBD patients. (A) $11.54 \%$ (UC) to $29.90 \%$ (CD) of IBD patients currently smoke in our patient cohort. (B) Smoking reduces QUOL significantly in CD and by trend in UC. The boxplot's median is depicted using a horizontal black line. Cutoff of the score for the QUOL was 60 points in the Short Inflammatory Bowel Disease Questionnaire (SIB$\mathrm{DO}$ ) and is depicted using a red line ( $\geq 60$ normal QUOL, $<60$ moderately to severely reduced QUOL). A $t$-test was used for statistical analysis. ${ }^{a} P<0.01$. IBD, inflammatory bowel disease; $U C$, ulcerative colitis; $C D$, Crohn's disease.

QUOL we found that DCCV membership is associated with a significantly higher QUOL in both subgroups (Table 1). In addition, due to the high prevalence of smoking in both UC patients (11.54\%) and CD patients (29.90\%) (Fig. 4) and the known effects of smoking on disease activity, we analyzed its effect on QUOL. Non-smoking is associated with a significantly improved QUOL in CD patients and by trend to improved QUOL in UC patients as well (Fig. 4).

Additional factors, with a positive influence on QUOL seem to be age and sex. The older the patients and the longer the duration of the disease (IBD in years), the better the QUOL becomes in both entities, whereas female sex in IBD is associated with a reduced QUOL (Table 1). Polypharmacy in terms if a higher number of prescribed pharmaceuticals is associated with a reduced QUOL, which means that our medications are not sufficient to relief all IBD associated health-related issues. High FoP (FoP-Q-SF <36) and a high demand for psychotherapy (ADAPT $\geq 60$ ) are associated with a reduced QUOL in both entities (Table 1).

\section{Predictors of QUOL in Patients with IBD (Whole Patient Cohort)}

As shown in Fig. 5, multiple stepwise linear regression analysis of all studied variables demonstrated non-smoking $(P=0.036)$, no IBD medication $(P=0.045)$, no EIM $(P<0.001)$, low FoP $(P<0.001)$ and low demand for psychotherapy $(P<0.001)$ were independent predictors of superior QUOL. Therefore, these 5 variables explain an improved QUOL in IBD patients, with the total model explaining $48.5 \%$ of the variance. 
Table 1. Patient Characteristics Regarding Quality of Life

\begin{tabular}{|c|c|c|c|c|c|c|}
\hline \multirow{3}{*}{ Characteristic } & \multicolumn{6}{|c|}{ Quality of life (SIBDQ score) } \\
\hline & \multicolumn{2}{|c|}{ IBD } & \multicolumn{2}{|c|}{ UC } & \multicolumn{2}{|c|}{$C D$} \\
\hline & Mean \pm SD & $P$-value ${ }^{a}$ & Mean \pm SD & $P$-value ${ }^{a}$ & Mean \pm SD & $P$-value \\
\hline Sex & & 0.009 & & 0.668 & & 0.003 \\
\hline Male & $45.0 \pm 12.3$ & & $44.8 \pm 12.8$ & & $45.6 \pm 11.8$ & \\
\hline Female & $42.0 \pm 12.2$ & & $44.0 \pm 12.4$ & & $40.9 \pm 12.1$ & \\
\hline Age (yr) & & 0.009 & & 0.055 & & 0.040 \\
\hline$\leq 30$ & $40.1 \pm 12.5$ & & $42.4 \pm 13.3$ & & $39.9 \pm 11.9$ & \\
\hline$>30$ & $43.7 \pm 12.2$ & & $45.7 \pm 11.9$ & & $42.7 \pm 12.3$ & \\
\hline Duration of the IBD (yr) & & $0.001^{b}$ & & $<0.001^{b}$ & & $0.317^{b}$ \\
\hline $0-5$ & $39.9 \pm 12.3$ & & $40.0 \pm 12.7$ & & $40.0 \pm 12.1$ & \\
\hline $6-10$ & $41.5 \pm 11.4$ & & $41.9 \pm 10.8$ & & $41.1 \pm 12.0$ & \\
\hline $11-15$ & $44.9 \pm 12.1$ & & $48.8 \pm 11.8$ & & $42.7 \pm 11.7$ & \\
\hline$>15$ & $44.5 \pm 12.6$ & & $48.6 \pm 12.1$ & & $42.9 \pm 12.4$ & \\
\hline No. of prescribed pharmaceuticals for IBD & & $<0.001$ & & $<0.001$ & & $<0.001$ \\
\hline None & $48.9 \pm 12.1$ & & $53.9 \pm 12.6$ & & $47.4 \pm 11.9$ & \\
\hline 1 & $45.0 \pm 12.2$ & & $45.4 \pm 12.1$ & & $44.8 \pm 12.3$ & \\
\hline $2-3$ & $42.2 \pm 11.7$ & & $44.9 \pm 11.5$ & & $40.4 \pm 11.5$ & \\
\hline$\geq 4$ & $36.3 \pm 11.9$ & & $36.8 \pm 13.5$ & & $36.1 \pm 11.0$ & \\
\hline Undergone IBD surgery & & 0.665 & & 0.911 & & 0.081 \\
\hline No & $42.5 \pm 12.8$ & & $44.1 \pm 12.8$ & & $40.4 \pm 12.5$ & \\
\hline Yes & $42.9 \pm 11.8$ & & $44.4 \pm 11.3$ & & $42.7 \pm 11.9$ & \\
\hline Psychotherapy experience & & 0.038 & & 0.060 & & 0.317 \\
\hline No & $43.7 \pm 12.3$ & & $45.7 \pm 12.3$ & & $42.4 \pm 12.2$ & \\
\hline Yes & $41.6 \pm 12.4$ & & $42.5 \pm 12.7$ & & $41.6 \pm 12.2$ & \\
\hline Extraintestinal manifestations & & $<0.001$ & & $<0.001$ & & $<0.001$ \\
\hline No & $47.1 \pm 11.4$ & & $47.3 \pm 11.6$ & & $47.0 \pm 11.3$ & \\
\hline Yes & $38.9 \pm 11.5$ & & $38.4 \pm 12.1$ & & $37.7 \pm 11.3$ & \\
\hline Smoking & & $<0.001$ & & & & 0.001 \\
\hline No & $43.7 \pm 12.4$ & & $44.5 \pm 12.7$ & & $43.1 \pm 12.1$ & \\
\hline Yes & $39.0 \pm 11.4$ & & $41.2 \pm 10.1$ & & $38.5 \pm 11.6$ & \\
\hline Stoma & & 0.029 & & 0.604 & & 0.014 \\
\hline No & $42.4 \pm 12.5$ & & $44.1 \pm 12.8$ & & $41.4 \pm 12.3$ & \\
\hline Yes, transient & $39.6 \pm 11.5$ & & $42.9 \pm 11.6$ & & $37.2 \pm 11.1$ & \\
\hline Yes, currently & $46.5 \pm 10.7$ & & $48.6 \pm 7.2$ & & $46.2 \pm 11.3$ & \\
\hline DCCV member & & $<0.001$ & & $<0.001$ & & 0.004 \\
\hline No & $40.0 \pm 12.6$ & & $40.6 \pm 13.4$ & & $39.6 \pm 12.2$ & \\
\hline Yes & $44.9 \pm 11.9$ & & $47.3 \pm 11.3$ & & $43.5 \pm 12.2$ & \\
\hline Fear of progression (FoP-Q-SF) & & $<0.001$ & & $<0.001$ & & $<0.001$ \\
\hline$\geq 36$ & $51.1 \pm 10.2$ & & $51.8 \pm 10.0$ & & $50.5 \pm 10.4$ & \\
\hline$<36$ & $37.1 \pm 10.3$ & & $37.2 \pm 10.3$ & & $37.0 \pm 10.4$ & \\
\hline Demand for psychotherapy (ADAPT) & & $<0.001$ & & $<0.001$ & & $<0.001$ \\
\hline$<60$ & $46.9 \pm 11.7$ & & $50.0 \pm 10.7$ & & $45.1 \pm 11.9$ & \\
\hline$\geq 60$ & $39.3 \pm 11.7$ & & $39.0 \pm 11.6$ & & $39.5 \pm 11.8$ & \\
\hline
\end{tabular}

${ }^{a}$ Student $t$-test.

${ }^{\circ}$ Analysis of variance.

SIBDQ, Short Inflammatory Bowel Disease Questionnaire; IBD, inflammatory bowel disease; UC, ulcerative colitis; CD, Crohn's disease; SD, standard deviation; DCCV, Deutsche Morbus Crohn/Colitis ulcerosa Vereinigung; Fop-Q-SH, Fear of Progression Questionnaire Short Form; ADAPT, Assessment of the Demand for Additional Psychological Treatment. 


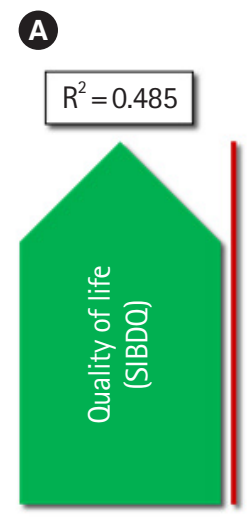

B
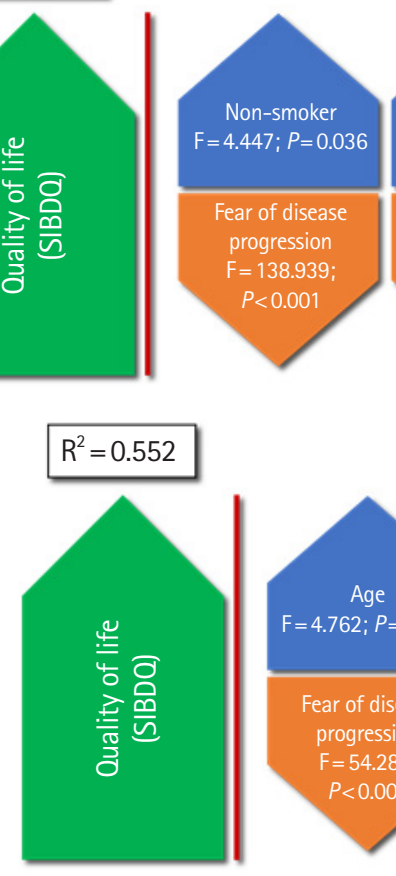

Whole cohort of IBD patients

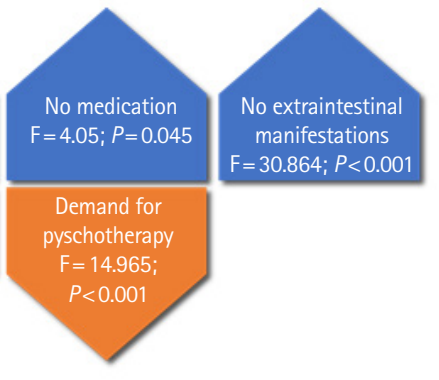

UC patients

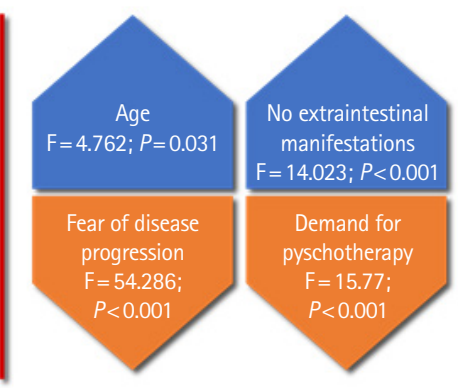

C

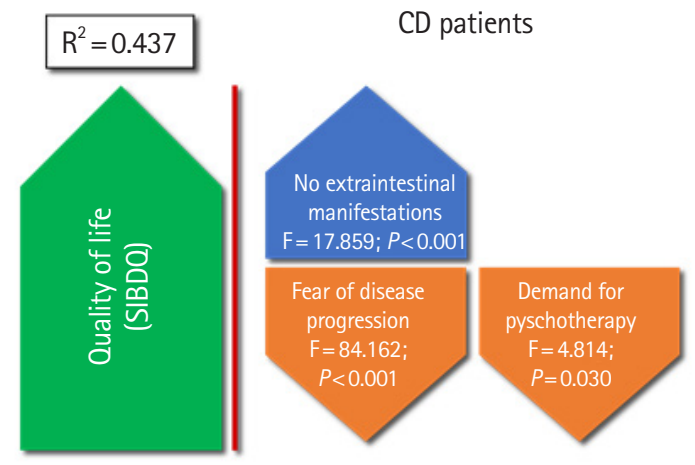

Fig. 5. Predictors of normal quality of life (QUOL) in IBD patients (whole cohort, UC patients and CD patients, respectively, defined by Short Inflammatory Bowel Disease Questionnaire [SIBDO] score $\geq 60$ ). Regression analyses of all IBD patients in our cohort (UC and $C D$ ) (A) and singular entities UC (B) and CD (C) reveal different variables predicting a normal QUOL. The direction of arrows indicates "positive" (up) or "negative" (down) association of the respective predictor with QUOL. In the case of dichotomous variables "yes" (up, presence of a factor) or "no" (down, absence of a factor) increases/decreases QUOL. IBD, inflammatory bowel disease; UC, ulcerative colitis; $C D$, Crohn's disease.

\section{Predictors of QUOL in Patients with UC and CD}

Superior QUOL in UC is associated with higher age $(P=0.031)$, no EIM $(P<0.001)$, low FOP $(P<0.001)$ and low demand for psychotherapy $(P<0.001)$. These predicting variables together explain $55.2 \%$ data variance (Fig. 5B). In CD, superior QUOL is associated with no EIM $(P<0.001)$, low FOP $(P<0.001)$ and low demand for psychotherapy $(P=0.030)$. Together, these 3 factors explain $43.7 \%$ of data variance (Fig. 5C).

\section{DISCUSSION}

In this study, $87.34 \%$ of UC patients and $91.08 \%$ of CD patients demonstrated an impaired QUOL, being in line with several other studies showing also diminished QUOL in various IBD patient cohorts: Williet et al. ${ }^{13}$ even evaluated a cohort where $100 \%$ of patients suffering from both entities scored a SIBDQ $<60$, showing an at least moderately impaired QUOL when using our cutoff. This suggests that our study does not contain a significant selection bias and proves an impaired QUOL to be a major issue in IBD patients with no difference between both entities; however, most analyses of the course of disease focus on intestinal manifestations (stool frequency, mucosal healing, etc.). In addition, active EIM are distributed through IBD cohorts between 35.93\% (UC) and 55.47\% (CD) (Fig. 2), but despite their high prevalence, EIM are often neglected in the evaluation of the course of disease.

In our cohort, EIM showed a significant impact on QUOL in both entities, which is also underlined in the regression analysis wherein EIM are one influence factor in the whole cohort as well as in the analysis for UC and CD, being the only factor beside FOP and ADAPT in the CD analysis. Therefore, our data seem to exert reliable information for the need to be aware of EIM control as therapeutic goal.

Furthermore, while it is a well-known fact that the creation of a stoma is a big fear for many patients, our analysis proves the creation of a stoma to be associated with an improved QU$\mathrm{OL}$ in CD patients, and with a trend in the same direction in UC patients (Fig. 3). This is in fact controversially discussed in the current literature, with studies mostly showing a negative impact or no impact of ostomies on QUOL. ${ }^{21-23}$ However, in our own clinical experience, stoma creation is often feared by patients beforehand, while disease control after stoma creation often is worth the intervention. Interestingly, patients who used to have a stoma and got it reversed showed similar or even lower QUOL scores when compared to patients who had not had a stoma. This may be due to the fact that after the (mostly patient-initiated and not medically necessary) stoma relocation, disease control may be worsened again, thus decreasing the QUOL. In conclusion, stoma relocation should be evaluated highly critical and patients must be consequently 
informed about possible recurrence of symptoms.

Smoking is a well-known negative factor, especially in CD. Despite this knowledge we were surprised to find about $30 \%$ of CD patients actively smoking with a significant negative impact on QUOL. However, this negative influence is not surprising, as smoking is associated with a more active $\mathrm{CD}_{,}{ }^{24}$ underlining the need for structured stop-smoking programs in this cohort. As is observable in the regression analysis, smoking is negatively influencing QUOL for the whole cohort.

The number of medications is not related to an improved, but instead to a diminished QUOL, indicating that our medications are not sufficient to help all patients with all aspects of the disease. However, patients with many medications often suffer from a higher disease activity resulting in a diminished QUOL and possibly causing a confounding error.

The significant association of FOP and demand for psychotherapy (ADAPT) with QUOL underlines that important aspects of a chronic disease are not sufficiently addressed by common activity scores focusing on somatic symptoms. In a previous analysis of this patient cohort we could find that psychotherapy experience and demand is a common and important topic for IBD patients. ${ }^{18}$

As a weakness of our study, we did not include clinical data, since all our data were derived from questionnaires completed by patients at home and collected via the internet; this may result in a degree of interpretation bias. However, since QUOL is also highly subjective, we judged this approach to be suitable.

In conclusion, EIM, smoking, high fear of disease progression and high demand for psychotherapy show a negative impact on QUOL. Stoma creation can improve QUOL, whereas polypharmacy cannot solve all problems related to IBD, but instead shows an association with impaired QUOL. With the aim to improve QUOL in IBD patients, thorough patient education concerning ostomies is important to reduce rejection of this potentially beneficial therapeutic step. Most importantly, physicians should not only focus on mucosal healing, stool frequency, and somatic disease markers such as EIMs, but as well on social and psychological factors around the disease.

\section{ADDITIONAL INFORMATION}

\section{Funding Source}

This study was supported by the Deutsche Forschungsgemeinschaft, Germany (Heisenberg Program).

\section{Conflict of Interest}

No potential conflict of interest relevant to this article was reported.

\section{Author Contribution}

Study concept and design: Keller R, Malek NP, Wehkamp J, Enck P, Klag T. Acquisition of data: Keller R, Fantasia L, Enck P, Klag T. Analysis and interpretation of data: Keller R, Mazurak N, Fantasia L, Fusco S, Enck P, Klag T. Drafting of the manuscript: Keller R, Enck P, Klag T. Critical revision of the manuscript for important intellectual content: Mazurak N, Fantasia L, Fusco S, Malek NP, Wehkamp J, Enck P, Klag T. Statistical analysis: Keller R, Mazurak N, Fantasia L, Enck P, Klag T. Obtained funding: Wehkamp J, Klag T. Technical, or material support: Wehkamp J, Enck P, Klag T. Study supervision: Malek NP, Wehkamp J, Enck P, Klag T. Approval of final manuscript: all authors.

\section{ORCID}

Keller R https://orcid.org/0000-0003-4023-1711

Klag T https://orcid.org/0000-0001-6286-1226

\section{Supplementary Material}

Supplementary materials are available at the Intestinal Research website (https://www.irjournal.org).

\section{REFERENCES}

1. Schreiber S, Hartmann H, Kruis W, et al. Inflammatory bowel disease competence network. Bundesgesundheitsblatt Gesundheitsforschung Gesundheitsschutz 2016;59:465-474.

2. Wehkamp J, Götz M, Herrlinger K, Steurer W, Stange EF. Inflammatory bowel disease. Dtsch Arztebl Int 2016;113:72-82.

3. Walsh AJ, Bryant RV, Travis SP. Current best practice for disease activity assessment in IBD. Nat Rev Gastroenterol Hepatol 2016;13:567-579.

4. Sandborn WJ, Feagan BG, Rutgeerts P, et al. Vedolizumab as induction and maintenance therapy for Crohn's disease. N Engl J Med 2013;369:711-721.

5. Annese V. A review of extraintestinal manifestations and complications of inflammatory bowel disease. Saudi J Med Med Sci 2019;7:66-73.

6. Masaki T, Kishiki T, Kojima K, Asou N, Beniya A, Matsuoka H. Recent trends (2016-2017) in the treatment of inflammatory bowel disease. Ann Gastroenterol Surg 2018;2:282-288.

7. Parkes GC, Whelan K, Lindsay JO. Smoking in inflammatory bowel disease: impact on disease course and insights into the 
aetiology of its effect. J Crohns Colitis 2014;8:717-725.

8. Irvine EJ, Zhou Q, Thompson AK. The Short Inflammatory Bowel Disease Questionnaire: a quality of life instrument for community physicians managing inflammatory bowel disease. CCRPT Investigators. Canadian Crohn's Relapse Prevention Trial. Am J Gastroenterol 1996;91:1571-1578.

9. Jowett SL, Seal CJ, Barton JR, Welfare MR. The Short Inflammatory Bowel Disease Questionnaire is reliable and responsive to clinically important change in ulcerative colitis. Am J Gastroenterol 2001;96:2921-2928.

10. Han SW, Gregory W, Nylander D, et al. The SIBDQ: further validation in ulcerative colitis patients. Am J Gastroenterol 2000;95:145-151.

11. Guyatt G, Mitchell A, Irvine EJ, et al. A new measure of health status for clinical trials in inflammatory bowel disease. Gastroenterology 1989;96:804-810.

12. Rose M, Fliege H, Hildebrandt M, et al. Validation of the new German translation version of the "Short Inflammatory Bowel Disease Questionnaire” (SIBDQ). Z Gastroenterol 2000;38: 277-286.

13. Williet N, Sarter H, Gower-Rousseau C, et al. Patient-reported outcomes in a French nationwide survey of inflammatory bowel disease patients. J Crohns Colitis 2017;11:165-174.

14. Miehsler W, Weichselberger M, Offerlbauer-Ernst A, et al. Assessing the demand for psychological care in chronic diseases: development and validation of a questionnaire based on the example of inflammatory bowel disease. Inflamm Bowel Dis 2004;10:637-645.

15. Miehsler W, Weichselberger M, Offerlbauer-Ernst A, et al. Which patients with IBD need psychological interventions? A controlled study. Inflamm Bowel Dis 2008;14:1273-1280.
16. Miehsler W, Dejaco C, Moser G. Factor analysis of ADAPT questionnaire for assessment of subjective need for psychological interventions. Inflamm Bowel Dis 2008;14:142-143.

17. Staufer K, Scherzer TM, Miehsler W, et al. Self-reported need for psychotherapy predicts interferon-induced depression in hepatitis C: stratification for interferon-free treatment. Antivir Ther 2015;20:501-506.

18. Klag T, Mazurak N, Fantasia L, et al. High demand for psychotherapy in patients with inflammatory bowel disease. Inflamm Bowel Dis 2017;23:1796-1802.

19. Mehnert A, Herschbach P, Berg P, Henrich G, Koch U. Fear of progression in breast cancer patients: validation of the short form of the Fear of Progression Questionnaire (FoP-Q-SF). Z Psychosom Med Psychother 2006;52:274-288.

20. Erim Y, Loquai C, Schultheis U, et al. Anxiety, posttraumatic stress, and fear of cancer progression in patients with melanoma in cancer aftercare. Onkologie 2013;36:540-544.

21. Knowles SR, Wilson J, Wilkinson A, et al. Psychological wellbeing and quality of life in Crohn's disease patients with an ostomy: a preliminary investigation. J Wound Ostomy Continence Nurs 2013;40:623-629.

22. Abdalla MI, Sandler RS, Kappelman MD, et al. The impact of ostomy on quality of life and functional status of Crohn's disease patients. Inflamm Bowel Dis 2016;22:2658-2664.

23. Dibley L, Czuber-Dochan W, Duncan J, et al. NO002 Decisionmaking about emergency and planned stoma surgery for IBD: a qualitative exploration of patient and clinician perspectives. J Crohns Colitis 2017;11(Suppl 1):S487-S488.

24. Cosnes J. Smoking, physical activity, nutrition and lifestyle: environmental factors and their impact on IBD. Dig Dis 2010; 28:411-417. 


\section{See "Quality of life in inflammatory bowel diseases: it is not all about the bowel" on page 45-52.}

Supplementary Table 1. Summarized Patient Characteristics of the Examined Cohort

\begin{tabular}{|c|c|c|c|}
\hline \multirow{2}{*}{ Characteristic } & \multicolumn{3}{|c|}{ No. of patients (\%) } \\
\hline & IBD & UC & $C D$ \\
\hline Patient data & $631(100)$ & 235 (37.2) & $393(62.3)$ \\
\hline \multicolumn{4}{|l|}{ Sex } \\
\hline Male & $159(25.4)$ & 77 (33.0) & $80(20.6)$ \\
\hline Female & $466(74.6)$ & $156(67.0)$ & $309(79.4)$ \\
\hline Total & 625 & 233 & 389 \\
\hline \multicolumn{4}{|l|}{ Relationship status } \\
\hline Single & $185(29.4)$ & $68(28.9)$ & $116(29.7)$ \\
\hline In a relationship & $213(33.9)$ & $80(34.0)$ & $132(33.8)$ \\
\hline Married & $231(36.7)$ & $87(37.0)$ & $143(36.6)$ \\
\hline Total & 629 & 235 & 391 \\
\hline \multicolumn{4}{|l|}{ Age (yr) } \\
\hline$\leq 30$ & 231 (37.3) & $92(40.4)$ & $139(35.7)$ \\
\hline$>30$ & $389(62.7)$ & $136(59.6)$ & $250(64.3)$ \\
\hline Total & 620 & 228 & 389 \\
\hline \multicolumn{4}{|c|}{ Duration of the IBD (yr) } \\
\hline$\leq 1$ & $37(6.1)$ & $11(4.8)$ & $25(6.6)$ \\
\hline $2-10$ & $271(44.7)$ & $119(52.4)$ & $151(40.2)$ \\
\hline$>10$ & $298(49.2)$ & $97(42.7)$ & $200(53.2)$ \\
\hline Total & 606 & 227 & 376 \\
\hline \multicolumn{4}{|c|}{ No. of prescribed pharmaceuticals for IBD } \\
\hline None & $45(7.1)$ & $10(4.3)$ & $34(8.7)$ \\
\hline $1-3$ & $482(76.4)$ & $189(80.4)$ & $293(74.6)$ \\
\hline$\geq 4$ & $104(16.5)$ & $36(15.3)$ & 66 (16.8) \\
\hline Total & 631 & 235 & 393 \\
\hline
\end{tabular}

No. of inpatient treatments (last $3 \mathrm{yr}$ )

$\begin{array}{lccc}\text { None } & 245(42.8) & 108(49.3) & 136(38.9) \\ 1-3 & 283(49.5) & 100(45.7) & 181(51.7) \\ \geq 4 & 44(7.7) & 11(5.0) & 33(9.4) \\ \text { Total } & 572 & 219 & 350\end{array}$

Undergone IBD-surgery

$\begin{array}{lccc}\text { No } & 314(54.6) & 202(86.0) & 166(42.2) \\ \text { Yes } & 261(45.4) & 33(14.0) & 227(57.8) \\ \text { Total } & 575 & 235 & 393\end{array}$

Psychotherapy experience

\begin{tabular}{lccc} 
No & $309(50.1)$ & $119(51.5)$ & $189(49.3)$ \\
Yes & $308(49.9)$ & $112(48.5)$ & $194(50.7)$ \\
Total & 617 & 231 & 383 \\
\hline
\end{tabular}

(Continued to the next)
Supplementary Table 1. Continued

\begin{tabular}{|c|c|c|c|}
\hline \multirow{2}{*}{ Characteristic } & \multicolumn{3}{|c|}{ No. of patients (\%) } \\
\hline & IBD & UC & $C D$ \\
\hline \multicolumn{4}{|c|}{ Extraintestinal manifestations } \\
\hline No & $320(51.8)$ & $148(64.1)$ & $171(44.5)$ \\
\hline Yes & $298(48.2)$ & $83(35.9)$ & $213(55.5)$ \\
\hline Total & 618 & 231 & 384 \\
\hline \multicolumn{4}{|l|}{ Smoking } \\
\hline No & $481(77.0)$ & 207 (88.5) & $272(70.1)$ \\
\hline Yes & $144(23.0)$ & $27(11.5)$ & $116(29.9)$ \\
\hline Total & 625 & 234 & 388 \\
\hline \multicolumn{4}{|l|}{ Stoma } \\
\hline No & $530(86.2)$ & $208(90.8)$ & $320(83.6)$ \\
\hline Yes, transient & $33(5.4)$ & $14(6.1)$ & $19(5.0)$ \\
\hline Yes, currently & $52(8.5)$ & $7(3.1)$ & $44(11.5)$ \\
\hline Total & 615 & 229 & 383 \\
\hline \multicolumn{4}{|l|}{ DCCV member } \\
\hline No & $224(39.8)$ & 77 (36.8) & $146(41.6)$ \\
\hline Yes & $339(60.2)$ & $132(63.2)$ & $205(58.4)$ \\
\hline Total & 563 & 209 & 351 \\
\hline \multicolumn{4}{|l|}{ Quality of life (SIBDO) } \\
\hline Impaired QUOL (<60) & $540(89.7)$ & $200(87.3)$ & $337(91.1)$ \\
\hline Normal QUOL ( $\geq 60$ ) & $62(10.3)$ & $29(12.7)$ & $33(8.9)$ \\
\hline Total & 602 & 229 & 370 \\
\hline \multicolumn{4}{|c|}{ Fear of progression (FoP-Q-SF) } \\
\hline Low FoP $(<36)$ & 240 (39.8) & $110(47.8)$ & $130(35.1)$ \\
\hline High FoP ( $\geq 36)$ & $363(60.2)$ & $120(52.2)$ & $240(64.9)$ \\
\hline Total & 603 & 230 & 370 \\
\hline \multicolumn{4}{|c|}{ Demand for psychotherapy (ADAPT) } \\
\hline Low demand $(\leq 60)$ & $256(44.3)$ & $93(42.5)$ & $162(45.5)$ \\
\hline High demand (>60) & $322(55.7)$ & $126(57.5)$ & $194(54.5)$ \\
\hline Total & 578 & 219 & 356 \\
\hline
\end{tabular}

IBD, inflammatory bowel disease; UC, ulcerative colitis; $C D$, Crohn's disease DCCV, Deutsche Morbus Crohn/Colitis ulcerosa Vereinigung; SIBDQ, Short Inflammatory Bowel Disease Questionnaire; QUOL, quality of life; FoP-QSH, Fear of Progression Questionnaire Short Form; ADAPT, Assessment of the Demand for Additional Psychological Treatment. 